\section{Impact of Incidental Findings on Neuroimaging Research Using Functional MR Imaging}

We read with great interest the recent article of Brown and Hasso, ${ }^{1}$ in which the authors discuss the handling of incidental findings in neuroimaging research. In our institution, Institutional Review Board (IRB) approval is obtained for all new functional MR imaging (fMRI) studies. Recently, a patient diagnosed with a large meningioma of the left sphenoid wing was referred to the Department of Neurosurgery of the University Hospital Schleswig-Holstein. The patient had previously been participating in a study measuring fat in various regions of the body, including the brain, with use of MR imaging. The lesion was clearly visible on these images; however, no radiologist or neuroradiologist was involved in the study, and thus the images were not read by a trained person at that time. On the basis of that case, our local IRB changed its policy, requiring that all MR images of the brain performed in research studies be read by a board-certified neuroradiologist.

A tremendous discussion arose in which various aspects of this problem were addressed and which ultimately resulted in a form of management that should cover all of the needs of subjects who will probably become patients, principal investigators (PIs), and involved neuroradiologists. Incidental findings are not uncommon, ${ }^{1-4}$ ranging from $0.2 \%$ to $1.6 \%$ for incidentally found brain tumors within the study population, or vascular abnormalities, ranging from $0.51 \%$ to $1.8 \%$. Both rates depend on the age of the subjects, which may thus have a major effect on the volunteers' lives.

Whereas volunteers are usually searched for and selected by placard, Webpage announcement, or word-of-mouth advertising within the academic institution, subjects of fMRI studies are often students or colleagues participating in fundamental research studies. To claim that an investigation includes healthy participants, these individuals are usually no older than 35 years.

Together with the local IRB, neuroimaging researchers, and neuroradiologists, a new means of handling informed consent, the neuroimaging report, and incidental findings in neuroimaging research was developed.

Before imaging, subjects must give their signed informed consent. In addition to the standard information and questions regarding MR imaging safety, they are also informed that there might be incidental findings such as tumors or vascular anomalies that might influence their future. They are told that, besides the possibility of therapy being required (almost) immediately, such findings might influence their professional career, insurance issues, and family planning, too, and findings will be documented from this point forward. If they do not sign this document, they are not eligible for any study. Volunteers who want to remain anonymous represent a major issue here. For example, when studies on sexual preferences including homosexual or even pedophilic subjects are performed, the volunteers do not want to be linked to or registered by the hospital administration.

To overlay fMRI data on high-resolution images, most investigators perform a 3D T1-weighted scan, such as a magnetization-prepared rapid acquisition of gradient echo sequence, in our institution performed in the sagittal view (in-plane resolution, $1 \times 1 \mathrm{~mm}$ ). That most PIs are not trained to read films, as stated by Brown and Hasso, ${ }^{1}$ raises an ethical problem in that findings might be missed, as demonstrated in the aforementioned case. Thus, we mutually agreed to add an axial fluid-attenuated inversion recovery sequence to the protocol; both scans are read by a board-certified neuroradiologist, and a radiology report is created. Obviously, the neuroradiologist thus takes the responsibility to detect and report incidental findings on healthy volunteers. However, these 2 sequences certainly cannot replace a routine MR imaging examination; therefore, that is additionally noted on the report. Patient findings are assessed, and suspect findings are immediately reported to the PI. If there are any suspect findings, the volunteers are seen in the outpatient clinic, and additional diagnostic study or therapy is initiated if necessary. As of now, this protocol was only required once in a young man with a left temporal cavernoma. He is now being followed and is excluded from the study population. In the anonymous group, subjects are instructed to call the PI after their scans have been read. If there is a suspect finding, they receive their anonymous data on a compact disc and are told to see their primary care physician or a specialist if they do not want to be enrolled at the local clinic.

This form of management covers all the needs of volunteers who would probably become patients, neuroradiologists reading the cases, and the usually untrained PIs to help them avoid missing pathologic findings and to take the responsibility and the consequences arising from participating in a neuroimaging study. Additional enrollment of many more volunteers is needed to judge satisfaction with this form of management in the long run, but we believe that it meets the ethical challenge of incidental findings arising in neuroimaging studies of healthy volunteers.

\section{References}

1. Brown DA, Hasso AN. Toward a uniform policy for handling incidental findings in neuroimaging research. AJNR Am J Neuroradiol 2008;29:1425-27

2. Vernooij MW, Ikram MA, Tanghe HL, et al. Incidental findings on brain MRI in the general population. $N$ Engl J Med 2007;357:1821-28

3. Weber $\mathrm{F}, \mathrm{Knopf} \mathrm{H}$. Incidental findings in magnetic resonance imaging of the brains of healthy young men. J Neurol Sci 2006;240:81-84

4. Katzman GL, Dagher AP, Patronas NJ. Incidental findings on brain magnetic resonance imaging from 1000 asymptomatic volunteers. JAMA 1999; 282:36-39

S. Ulmer

U.R. Jensen

O. Jansen

Institute of Neuroradiology

H.M. Mehdorn

Department of Neurosurgery Institutional Review Board

J. Schaub

Institutional Review Board

G. Deuschl

H.R. Siebner

Department of Neurology

University Hospital of Schleswig-Holstein

Kiel, Germany

DOI 10.3174/ajnr.A1383 